\title{
Impact of integrated genetic information on diagnosis and prognostication for myeloproliferative neoplasms in the next-generation sequencing era
}

\section{Jong-Mi Lee}

College of Medicine, Catholic University of Korea

Howon Lee

College of Medicine, Catholic University of Korea

Ki-Seong Eom

Seoul St. Mary's Hospital, The Catholic University of Korea

\section{Sung-Eun Lee}

Seoul St. Mary's Hospital, The Catholic University of Korea

Myungshin Kim ( $\nabla$ microkim@catholic.ac.kr)

College of Medicine, Catholic University of Korea https://orcid.org/0000-0001-8632-0168

Yonggoo Kim ( $\nabla$ yonggoo@catholic.ac.kr)

College of Medicine, Catholic University of Korea

\section{Research Article}

Keywords: Next generation sequencing, Myeloproliferative neoplasm, Diagnosis, Prognosis, Risk stratification

Posted Date: December 1st, 2020

DOI: https://doi.org/10.21203/rs.3.rs-112499/v1

License: (c) (i) This work is licensed under a Creative Commons Attribution 4.0 International License. Read Full License 


\section{Abstract}

Background: Since next-generation sequencing has been widely used in clinical laboratory, diagnosis and risk stratification of hematologic malignancies are greatly dependent on genetic aberrations.

Methods: In this study, we analyzed the genomic landscape of 200 patients with myeloproliferative neoplasms (MPNs) using targeted panel sequencing covering including 86 genes. Conventional BM karyotyping was also performed to determine chromosomal aberration. We analyzed relationships between genetic profiles and clinical outcomes including acute transformation, bone marrow fibrosis and death.

Results: Mutations in JAK2, CALR, and MPL were detected in $76.4 \%$ of MPNs. The proportion of patients with clonal genetic markers increased up to $86.4 \%$ when all detectable genetic aberrations were included. Significant co-occurring genetic aberrations potentially associated with phenotype and/or disease progression, including those in JAK2/SF3B1 ( $P=0.017)$ and TP53/del(13q), del(5q), $-7 / \operatorname{del}(7 q)$ and complex karyotypes ( $P=0.038, P<0.001, P<0.001$ and $P<0.001$, respectively) were detected. We also identified genetic aberrations associated with patient outcomes: TP53 and $-7 / \mathrm{del}(7 \mathrm{q})$ for inferior survival (HR, 95\% Cl: 64.2, 3.8-1096.5, $P=0.0041, \mathrm{HR}, 95 \% \mathrm{Cl}: 14.0,1.5-132.7, P=0.0219), R U N X 1, T P 53$ and IDH1/2 for leukemic transformation (HR, 95\% Cl: 68.1, 3.6-1300.4, $P=0.005, \mathrm{HR}, 95 \% \mathrm{Cl}: 16.3,1.2-222.7, P=0.0364$, $\mathrm{HR}, 95 \% \mathrm{Cl}: 32.5,2.8-371.1, P=0.0051), S F 2 B 1, I D H 1 / 2, A S X L 1$ and del(20q) for fibrotic progression (HR, 95\% Cl: 31.5, 4.1-243.3, $P=0.0009, \mathrm{HR}, 95 \% \mathrm{Cl}: 21.2,3.4-132.2, P=0.0011, \mathrm{HR}, 95 \%$ Cl: 4.3, 1.1-16.4, $P=0.0358, \mathrm{HR}, 95 \% \mathrm{Cl}: 44.5,6.1-323.0, P=0.0002)$. We compared risk stratification systems and found that mutation-enhanced prognostic scoring systems could identify lower risk polycythemia vera and essential thrombocythemia and higher risk primary myelofibrosis $(P<0.001, P<0.001$ and $P<0.001$, respectively). Furthermore, the new risk stratification systems showed better predictive capacity of patient outcome.

Conclusions: These results collectively indicate that integrated genetic information can enhance diagnosis and prognostication in patients with myeloproliferative neoplasms.

\section{Background}

Myeloproliferative neoplasms (MPNs) are characterized by clonal proliferation of hematopoietic cells that are fully differentiated and functional. MPNs are mainly classified into polycythemia vera (PV), essential thrombocythemia (ET), and primary myelofibrosis (PMF) according to disease manifestations. Some rare disease categories such as chronic neutrophilic leukemia (CNL) and chronic eosinophilic leukemia are also considered MPNs, in addition to unclassified MPNs. Mutations in three well-known driver genes, $J A K 2, C A L R$, and MPL, are used to diagnose PV, ET, and PMF. Recently, CSF3R was identified as a driver gene in CNL[1, 2]. JAK2 mutations have been detected in $90 \%$ to $95 \%$ of PV patients and $50 \%$ to $60 \%$ of ET and PMF patients. CALR mutations are the second most common mutation in MPNs, and are found in $20 \%$ to $25 \%$ of ET and $25 \%$ to $30 \%$ of PMF patients. MPL mutations are found in $3 \%$ of ET patients and 
$5 \%$ of PMF patients[3]. Patients without mutations in any of these driver genes can be diagnosed with ET or PMF when their clinical and hematologic features meet specific criteria, including clonal mutations in any of the following genes: ASXL1, EZH2, TET2, IDH1/2, SRSF2, and SF3B1 [1]. Knowledge of the mutational landscape not only facilitates objective diagnosis of MPN, but can also potentially be used as a prognostic factor. This area of research has benefited from application of next-generation sequencing (NGS) technology in clinical laboratories as well as research laboratories. Important gene mutations associated with patient outcomes, such as mutations in TP53 and ASXL1, have been identified using NGS approaches.[4-8]

Risk stratification of MPNs is based on clinical and hematologic features. Advanced age, leukocytosis, and venous thrombosis are considered risk factors for survival in PV [9] and ET [10]. PMF-specific risk models such as the International Prognostic Scoring System (IPSS) [11], Dynamic IPSS (DIPSS) [12], and DIPSS-plus, which includes cytogenetic abnormalities in addition to DIPSS, have been developed [13]. Results of recent studies indicate that gene mutations are significant prognostic factors in MPN and mutation-enhanced prognostic systems for MPNs have been introduced [14-16]. These systems can be used to determine risk for survival as well as disease progression, including leukemic transformation and fibrotic progression[17, 18]. Moreover, integration of genetic information with clinical variables can enable prediction of patient outcomes and allow development of personalized therapeutic plans based on consideration of causal biologic mechanisms [19-21]. It is challenging at least in the present moment because data are limited to make consensus across the ethnics and counties under different clinical environments.

In this study, we analyzed genetic aberrations using cytogenetic and molecular genetic methods, including NGS, to reveal their impact on the diagnosis of MPNs in current era. We also tried to evaluate their contributions for risk stratification for survival as well as disease progression

\section{Methods}

\section{Patients and samples}

Among patients diagnosed with Philadelphia-negative MPN and treated at Seoul St. Mary's Hematology Hospital, a total of 200 patients who requested an NGS study or available DNA samples for NGS analysis were included. MPN diagnosis and their subtypes were strictly re-evaluated and classified based on the 2017 WHO classifications.[22] Demographics and clinical and hematologic features, including outcome data, were reviewed retrospectively from medical records. Most DNA samples were from bone marrow (BM) aspirates taken at the time of diagnosis, but some were collected at the time of symptom aggregation during observation or the course of treatment. This study was carried out in accordance with the Declaration of Helsinki and approved by the Institutional Review Board of Seoul St. Mary's Hospital (KC20RISI0371). This retrospective study involves only minimal risk to the participants, so the IRB waived the requirement to obtain informed consent from patients.

\section{Molecular analysis}


DNA was extracted using the QIAamp DNA Mini Kit (Qiagen, Hilden, Germany). DNA concentration and purity were assessed using an ND-1000 spectrophotometer (Nanodrop Technologies, Wilmington, DE, USA). NGS was performed using a customized myeloid panel, the "SM panel", as described in our previous report [23]. Briefly, the SM panel contains 87 genes frequently mutated in patients with MPN (Supplemental Table 1). Target capturing sequencing was performed using a customized target kit (Agilent Technologies, 3039061) according to the manufacturer's instructions. DNA libraries were constructed according to the protocol provided by the manufacturer and sequencing was performed on an Illumina HiSeq4000 platform.

Sequenced reads were mapped to the human reference genome (hg19, Genome Reference Consortium, Feb 2009) using Burrows-Wheeler aligner. To call variants, we used VarScan v.2.3.9 with mpileup2snp and mpileup2indel. SnpEff v.4.2 was used to select variants located in coding sequences and predict their functional consequences. Annotated variants were further classified into four tiers according to the standards and guidelines of the Association for Molecular Pathology (AMP)[24]. All variants with a minor allele frequency $>0.01$ were filtered out based on the Exome Aggregation Consortium (ExAC, http://exac.broadinstitute.org/) and genome aggregation database (gnomAD, https://gnomad.broadinstitute.org/), as well as the ethnic-specific Korean Variant Archive (KOVA, http://kobic.re.kr /kova/). Variants of synonymous, intronic, or non-coding regions were further excluded. Among the remaining variants, variants with more than 20 reads and $5 \%$ variant allele frequencies (VAF) were considered to be mutations. Canonical mutations in JAK2, CALR, and MPL less than $5 \%$ VAF were considered to be present with a low allele burden. All mutations were manually verified using the Integrative Genomic Viewer.

\section{Cytogenetic study}

A total of 169 BM karyotypes were available. Conventional BM karyotyping was performed on G-banded metaphase chromosomes using routine techniques. Karyotypes were interpreted according to ISCN 2016 [25]. The number of cytogenetic events was considered the sum of chromosome gains, losses, partial gains, partial losses, and rearrangements.

\section{Statistical analyses}

Group comparisons according to phenotype were performed using the Kruskal-Wallis test and Chi-square test. Mann-Whitney test and Fisher's exact test were also used for post-hoc analysis. The significance of co-occurrences of mutations and chromosomal abnormalities was determined using Fisher's exact test.

The Chi-square test was used to assess whether the distributions of risk scores from two systems were systematically different. We defined overall survival (OS) as time from diagnosis to death from any cause, and event-free survival (EFS) as time from diagnosis to leukemic transformation, myelofibrosis progression, or death. Medical record tracking was done until February 2020. The Kaplan-Meier Log-rank test was used to estimate OS and EFS in each groups. Cox proportional-hazards regression was used for 
univariate and multivariate analyses. Statistical analyses were performed using MedCalc version 19.1.7 (MedCalc software, Mariakerke, Belgium).

\section{Results}

\section{Genetic landscape of MPNs}

Patients' clinical and hematologic characteristics are summarized in Table 1. According to disease category of WHO classification, ET was the largest group $(n=70,35.0 \%)$, followed by PMF $(n=66$, 33.0\%), PV ( $n=55,27.5 \%)$, and other MPNs $(n=9,4.5 \%)$ consisting of chronic neutrophilic leukemia (CNL, $n=4,2.0 \%)$ and MPN-unclassifiable $(n=5,2.5 \%)$. Age at diagnosis differed significantly according to disease category. ET patients (43.2 \pm 15.3 years) were younger than PV (50.3 \pm 12.3 years), PMF (52.5 \pm 13.6 years), and other MPN (61.4 \pm 14.2 years) patients $(P=0.0034, P=0.0002$ and $P=0.0024$, respectively). Clinical and genetic variables according to disease category are described in Supplemental Figure. 1.

Genetic landscapes for all patients are shown in Figure 1A. Mutations were identified in $85.5 \%$ of patients $(n=171)$. The mean number of mutations was $1.3 \pm 1.0$, and ET patients harbored significantly fewer mutations than PMF patients $(1.1 \pm 0.9$ vs. $1.6 \pm 1.1, P=0.024)$. JAK2 mutations $(\mathrm{n}=98)$ were the most common followed by CALR, ASXL1, TET2, and MPL mutations ( $\mathrm{n}=43,24,17$, and 5 , respectively). Abnormal karyotypes were identified in $22.5 \%$ of patients (38/169), and $4.7 \%(8 / 169)$ of individuals had a complex karyotype with three or more cytogenetic abnormalities. The proportion of abnormal karyotypes in PMF patients $(37.5 \%, 21 / 56)$ was higher than that in PV $(18.6 \%, 8 / 43)$ or ET $(9.8 \%, 6 / 61)$ patients $(P=$ $0.047, P=0.0004$, respectively). $-5 / \operatorname{del}(5 q)(n=10)$ was the most common cytogenetic abnormality, followed by del $(20 q)(n=9)$, del $(13 q)(n=9)$, and $-7 / \operatorname{del}(7 q)(n=6)$ (Figure 1B).

We also analyzed the co-occurrence of mutations and abnormal karyotypes. Multiple pairs of cooccurring mutations were found, including JAK2 with SF3B1, ASXL1 with SRSF2, and DNMT3A with IDH1/2 $(P=0.017, P=0.01$, and $P=0.012$, respectively). TP53 mutations were significantly associated with del(13q), -5/del(5q), -7/del(7q), and complex karyotypes $(P=0.038, P<0.001, P<0.001$ and $P<$ 0.001 , respectively) (Figure 1C) [26]. We reviewed Prussian blue iron staining of available BM samples with $J A K 2 / S F 3 B 1$ co-mutations and found ring sideroblasts in all three samples.

\section{Impact of genetic aberrations on diagnosis}

JAK2 $(\mathrm{n}=98,49.0 \%), \operatorname{CALR}(\mathrm{n}=43,21.5 \%)$, and MPL $(\mathrm{n}=5,2.5 \%)$ mutations were detected in $76.4 \%$ of $\mathrm{PV}, \mathrm{ET}$, and PMF patients $(\mathrm{n}=146)$. JAK2 mutations were detected in 49 PV patients $(89.1 \%)$ while JAK2, CARL, and MPL mutations were detected in 26 (37.1\%), 22 (31.4\%), and 3 (4.3\%) ET patients, respectively. JAK2, CARL, and MPL mutations were detected in 23 (34.8\%), 21 (31.8\%), and 2 (3.0\%) PMF patients, respectively. Among 39 triple-negative ET and PMF patients, 8 (2 ET and 6 PMF) patients had any of myeloid neoplasm-associated mutations in ASXL1, EZH2, TET2, IDH1/2, SRSF2, or SF3B1. CSF3R T618I mutation was detected in 2 of $4 \mathrm{CNL}$ patients. Thus, clonal genetic markers for diagnosis according the 
WHO classification were present in $80.6 \%(n=154)$ of three MPN as $89.1 \%$ of PV patients, $75.7 \%$ of ET patients, $78.8 \%$ of PMF patients, and $50 \%$ of CNL patients, representing $80.6 \%(n=154)$ of all MPN patients.

We considered all gene mutations, including those not mentioned above, in addition to an abnormal karyotype, as clonal genetic markers. Genetic aberrations were detected in 12 patients including mutations of U2AF1, DNMT3A, RUNX1, TP53, ZRSR2, KDM2B, NRAS, and KIT genes as well as karyotype abnormalities such as del(20q) and $-5 / \operatorname{del}(5 q)$. The proportion of MPN patients with clonal genetic markers increased up to $86.4 \%(n=165)$. When broken down according to MPN type, $90.9 \%$ of PV patients, $77.1 \%$ of ET patients, and $92.4 \%$ of PMF patients had clonal genetic markers (Table 2).

\section{Prognostic impact of genetic aberrations}

The mean overall survival of the study population was 400.5 months with a $95 \%$ confidence interval (Cl) of 367.0-434. 1 months. Fourteen deaths were recorded in the whole series (3 ET, 7 PMF and 4 other MPN). Clinical outcome in terms of shortest OS was significantly poorer in other MPN groups (36.8 months, 95\% Cl, 21.9-51.6) than the PV group (325.0 months, 95\% Cl, 325.0-325.0), ET group (296.4 months, 95\% Cl, 272.0-320.7), or PMF group (318.8 months, 95\% Cl, 218.8-418.7) (Figure 2A). Univariate analysis showed that mutations in TP53,-5/del(5q), -7/del(7q), del(20q), del(13q), number of abnormal karyotypes, and a complex karyotype were associated with poor outcomes. Diagnosis of PMF and presence of bone marrow fibrosis were also associated with poor outcomes. Hemoglobin level and JAK2 mutation were associated with a favorable outcome (Figure 2B). Next, we investigated the impact of clinical and hematologic variables and genetic aberrations on disease progression, including leukemic transformation and fibrotic progression. Nine patients experienced leukemic transformation (2 PV, 3 ET and 4 PMF), and there was no difference in frequency between the three disease groups. IDH1/2, RUNX1, and TP53 mutations, $-5 / \operatorname{del}(5 q),-7 / \operatorname{del}(7 q)$, number of abnormal karyotypes, and a complex karyotype were significant risk factors for leukemic transformation (Figure 2C). Fibrotic progression occurred in 24 (8 PV and $16 \mathrm{ET}$ ) patients. ASXL 1, IDH1/2, and SF3B1 mutations, number of mutations, and del(20q) were associated with fibrotic progression (Figure 2D). Details of the prognostic impact of each parameter are summarized in Supplemental Table 2.

In addition, we performed multivariate analysis. TP53 mutation, $-7 / \mathrm{del}(7 \mathrm{q})$, and diagnosis of PMF were adverse survival factors. PB blasts counts in addition to $R U N X 1, I D H 1 / 2$, and TP53 mutations were identified as risk factors for leukemic transformation. $A S X L 1, S F 3 B 1$, and IDH1/2 mutations, number of mutations, and del(20q) were defined as risk factors for fibrotic progression (Table 3). We also investigated the prognostic impact of genetic aberrations in each disease category. Despite limited numbers of patients in each disease category and duration, genetic aberrations identified as risk factors by multivariate analysis had statistical significance in log rank analysis (Supplemental Table 3).

\section{Impact of genetic aberrations on risk stratification}


To evaluate the impact of genetic aberrations on risk stratification, we compared the risk of each patient group before and after applying genetic aberrations. Although there were statistically significant relationships between risk groups (contingence coefficient 0.514 - 0.554), we found some interesting changes. Thirty-seven of $55 \mathrm{PV}$ patients had more than one risk factor including advanced age, leukocytosis, and a vascular event [9]. When adjusting for MIPSS-PV, which included SRSF2 mutation, 20 patients (54.1\%) were reclassified into the low risk group. In ET, 28 patients had more than one risk factor including advanced age, leukocytosis, and a vascular event [10]. When applying MIPSS-ET, which included SF3B1, SRSF2, TP53, and U2AF1 mutations, the majority of patients $(67.9 \%, 19 / 28)$ were reclassified as being low risk (Table 4). Among patients with PMF, 25 were classified as DIPSS low risk, 16 as intermediate risk, 21 as intermediate-2, and four as high risk $(n=4)$. It is notable that 10 and 6 patients in the low risk group were reclassified into intermediate and high risk groups based on MIPSS70[14] and MIPSS70+[27], respectively. MIPSS70 includes ASXL1,EZH2, SRSF2, and IDH1/2 mutations, while MIPSS70+ includes ASXL1, EZH2, SRSF2, and IDH1/2 mutations and cytogenetic risk categories. Three patients in intermediate-1 risk group were reclassified into high and very high risk groups by MIPSS70 and MIPSS70+, respectively (Table 5). It meant that the newly developed risk stratification systems including genetic aberrations discriminated more patients with low risk in PV and ET. On the other hand, the new systems selected PMF patient with higher risk among those with low or intermediate-1 risk.

Additionally, we analyzed the prediction capacity of mutation-enhanced prognostic scoring systems. In ET, MIPSS-ET revealed significant prediction of OS $(P=0.003)$ while the previous risk factors did not $(P=$ 0.922). In PMF, MIPSS70+ $(P=0.003)$ and MIPSS70 $(P=0.006)$ had a lower $P$ value for prediction of OS than DIPSS $(P=0.024)$. MIPSS70+ $(P=0.002)$ and MIPSS70 $(P=0.005)$ predicted EFS significantly better than DIPSS $(P=0.201)$ (Supplementary Table 4).

\section{Discussion}

Continuous advances in genetic technology have resulted in great changes in clinical practice. As a consequence of the advance, current diagnoses and classifications of hematologic malignancies are largely based on genetic aberrations. Diagnosis of MPNs is made primarily based on clinical and hematologic features supported by mutations in JAK2, CALR, and/or MPL genes. In the absence of mutations in any of these three genes, other myeloid neoplasm-associated mutations are recognized as another clonal markers for ET and PMF.[1] In the current study, we identified the driver mutations in $76.4 \%$ of MPN patients, with the highest frequency in PV patients (89.1\%) followed by ET (72.8\%) and PMF $(69.7 \%)$ patients. There are no clear definitions of myeloid neoplasm-associated mutations other than those in seven genes in the WHO classification. We found that gene mutations other than the three driver genes and cytogenetic abnormalities accounted for a considerable proportion of cases. When applying 7 myeloid neoplasm associated genes proposed by WHO classification, 8 patients became to possess clonal genetic markers. When including all detectable genetic aberrations, 12 patients were added to the list of patients with clonal genetic markers, and the proportion of patients with clonal genetic markers increased by $10.0 \%$. The added mutations and karyotype abnormalities were commonly detected in 
myeloid neoplasms or other malignancies such as U2AF1, DNMT3A, RUNX1, TP53, del(20q) and $-5 /$ del(5q). Therefore, it is necessary to define specific gene mutations and karyotype abnormalities and to clarify their role in the diagnosis of MPN.

Overall genetic landscapes were similar to those reported in previous studies.[28, 29] We found significant co-occurring mutations. JAK2/SF3B1 co-mutations, which are known to be associated with myelodysplastic/myeoproliferative neoplasms with ring sideroblasts and thrombocytosis (MDS/MPN-RST), were identified in 6 patients (2 ET and 4 PMF) [30, 31]. It is notable that ring sideroblasts were detected in all available BM samples with JAK2/SF3B1 co-mutations. Even though the proportion of RS was far less than 5\%, it was evident that the SF3B1 mutation affected the formation of RS. Further studies should determine an appropriate diagnostic strategy for this disease, which has a broad spectrum of phenotypes. TP53 mutation, co-occurring with del(13q), $-5 / \operatorname{del}(5 q),-7 / \operatorname{del}(7 q)$, and a complex karyotype, was a significant adverse survival factor. Studies have shown that TP53 mutations occur at a low frequency in chronic-phase MPNs and increase significantly according to disease progression. TP53 mutations were positively correlated with poor survival outcomes, including early death [4, 5, 21], and $-7 / \operatorname{del}(7 q)$ was associated with poor survival in PMF patients [32]. Because all patients with TP53 mutations had a complex karyotype, we postulated that presence of TP53 mutation resulted from accumulation of genetic aberrations through disease progression.

In addition, we identified genetic aberrations associated with disease progression. Results from this and previous studies showed that IDH1/2 mutations are associated with risk of leukemic transformation. IDH1/2 mutations occurred frequently in the blast phase of MPN compared to the chronic phase, suggesting a pathogenetic contribution to leukemic transformation [33]. This is worth noting because IDH inhibitors may offer a therapeutic advantage in high-risk MPN patients [34]. We also found that RUNX1 mutations were predictive of leukemic transformation. This finding is consistent with previous studies that demonstrated that $R U N X 1$ mutations had adverse impacts on leukemia-free survival in PV and ET patients $[16,17]$ and that $R U N X 1$-mutated PMF patients had inferior overall survival and leukemia-free survival [35]. Another important gene was ASXL 7; mutations in this gene are typically associated with fibrotic progression. $A S X L 1$ was the most frequently mutated gene with the exception of the three driver genes. Previous studies showed that $A S X L 1$ mutations were enriched for in patients with PMF [29], and ASXL 1 mutations are considered to be high-risk mutations along with mutations in epigenetic regulators such as SRSF2 and EZH2 in patients with MPNs $[8,17,28,36]$. TET2 is an epigenetic regulator frequently mutated in all MPN subtypes. Although previous studies have demonstrated that TET2 mutations increase the self-renewal capacity of hematopoietic stem cells [37] and can lead to disease initiation and progression [6], the prognostic value of TET2 mutations is controversial [7, 38]. Our study revealed that TET2 mutations were frequently detected in MPNs with little prognostic impact. Del(20q) was another significant factor associated with fibrosis progression. It is notable that most instances of del(20q) were identified in PMF patients or patients with PV or ET with fibrosis progression during follow-up.

Clinicians should stratify each patient's risk on the basis of updated information. This is even more important in the current era due to the increased availability of therapeutic options. Recent updates 
included genetic abnormalities into risk stratification for MPNs; SRSF2 for PV, SF3B1, SRSF2, TP53 and U2AF1 for ET, and ASXL1,EZH2, SRSF2, IDH1/2 and karyotype for PMF. It was expected that risk groups were correlated before and after applying genetic abnormalities, however, there were changes worth paying attention. In PV and ET, mutation-enhanced risk stratification system appeared to select low risk patients among patients with clinical and laboratory risk variables. By contrast, in PMF, mutation and additional karyotype-enhanced risk stratification systems identified higher risk patients among low or intermediate-1 risk groups, as supported by a previous finding that demonstrated the significance of ASXL 1 and SRSF2 mutations for treatment decision-making in low or intermediate-1 risk patients with PMF [39].

Due to the restricted number of patients in each disease category and relatively short follow-up period, further studies should be performed in a larger cohort considering racial heterogeneity. However, this study highlights the usefulness the diagnostic and prognostic potential of targeted sequencing in the NGS era. In addition, the results of our contemporary study can be used as valuable source to tailor therapeutic plans. Integrated genetic information not only facilitates diagnosis and prognostic expectations but also our understanding of the presentation and progression of MPNs.

\section{Conclusions}

Genetic landscape demonstrated that $86.4 \%$ of MPN patients harbored any clonal markers including $76.4 \%$ of MPN patients with triple driver mutations. Some genetic aberrations such as TP53, IDH1/2, SF3B1, RUNX1, -7/del(7q) and del(20q) were related to overall survival and/or disease progression, and mutation-enhanced scoring systems performed better than phenotype-based risk stratification. These findings support that integrated genetic information has a significant impact on the diagnosis and prognostication of MPN patients.

\section{Declarations}

\section{Ethics approval and consent to participate}

This study was carried out in accordance with the Declaration of Helsinki and approved by the Institutional Review Board of Seoul St. Mary's Hospital (KC20RISI0371). This retrospective study involves only minimal risk to the participants, so the IRB waived the requirement to obtain informed consent from patients.

\section{Consent for publication}

All authors agreed to publish.

\section{Availability of data and materials}


The datasets used and/or analysed during the current study are available from the corresponding author on reasonable request.

\section{Competing interests}

The authors declare that they have no competing interests.

\section{Funding}

Not applicable

\section{Authors' contributions}

M.S.K. and Y.G.K. designed research; J.M.L. and H.W.L. performed research and analyzed and interpreted data; K.S.E. and S.E.L performed validation and proofreading; J.M.L., M.S.K. and Y.G.K. wrote the manuscript. All authors have read and agreed to the published version of the manuscript.

\section{Acknowledgements}

This research was supported by the Bio \& Medical Technology Development Program of the National Research Foundation (NRF) funded by the Ministry of Science \& ICT (2020M3A9E8020866).

\section{References}

1. C.E. SS, NL H, ES J, SA P, H S, J T. WHO classification of tumours of haematopoietic and lymphoid tissues. 2017; Volume 2:104-105.

2. Pardanani A, Lasho T, Laborde R, et al. CSF3R T618I is a highly prevalent and specific mutation in chronic neutrophilic leukemia. Leukemia. 2013;27(9):1870-1873.

3. Jang M-A, Choi CW. Recent insights regarding the molecular basis of myeloproliferative neoplasms. The Korean Journal of Internal Medicine. 2020;35(1):1.

4. Marcellino BK, Hoffman R, Tripodi J, et al. Advanced forms of MPNs are accompanied by chromosomal abnormalities that lead to dysregulation of TP53. Blood advances. 2018;2(24):35813589 .

5. McNamara CJ, Panzarella T, Kennedy JA, et al. The mutational landscape of accelerated-and blastphase myeloproliferative neoplasms impacts patient outcomes. Blood advances. 2018;2(20):26582671.

6. Kameda T, Shide K, Yamaji T, et al. Loss of TET2 has dual roles in murine myeloproliferative neoplasms: disease sustainer and disease accelerator. Blood. 2015;125(2):304-315.

7. Abdel-Wahab O, Tefferi A, Levine RL. Role of TET2 and ASXL1 mutations in the pathogenesis of myeloproliferative neoplasms. Hematology/Oncology Clinics. 2012;26(5):1053-1064.

8. Guglielmelli P, Lasho TL, Rotunno G, et al. The number of prognostically detrimental mutations and prognosis in primary myelofibrosis: an international study of 797 patients. Leukemia. 
2014;28(9):1804-1810.

9. Tefferi A, Rumi E, Finazzi G, et al. Survival and prognosis among 1545 patients with contemporary polycythemia vera: an international study. Leukemia. 2013;27(9):1874-1881.

10. Passamonti F, Thiele J, Girodon F, et al. A prognostic model to predict survival in 867 World Health Organization-defined essential thrombocythemia at diagnosis: a study by the International Working Group on Myelofibrosis Research and Treatment. Blood. 2012;120(6):1197-1201.

11. Cervantes F, Dupriez B, Pereira A, et al. New prognostic scoring system for primary myelofibrosis based on a study of the International Working Group for Myelofibrosis Research and Treatment. Blood, The Journal of the American Society of Hematology. 2009;113(13):2895-2901.

12. Passamonti $F$, Cervantes $F$, Vannucchi AM, et al. A dynamic prognostic model to predict survival in primary myelofibrosis: a study by the IWG-MRT (International Working Group for Myeloproliferative Neoplasms Research and Treatment). Blood, The Journal of the American Society of Hematology. 2010;115(9):1703-1708.

13. Gangat N, Caramazza D, Vaidya R, et al. DIPSS plus: a refined Dynamic International Prognostic Scoring System for primary myelofibrosis that incorporates prognostic information from karyotype, platelet count, and transfusion status. Journal of Clinical Oncology. 2011;29(4):392-397.

14. Guglielmelli P, Lasho TL, Rotunno G, et al. MIPSS70: mutation-enhanced international prognostic score system for transplantation-age patients with primary myelofibrosis. Journal of Clinical Oncology. 2018;36(4):310-318.

15. Tefferi A, Guglielmelli P, Nicolosi M, et al. GIPSS: genetically inspired prognostic scoring system for primary myelofibrosis. Leukemia. 2018;32(7):1631-1642.

16. Tefferi A, Guglielmelli P, Lasho TL, et al. Mutation-enhanced international prognostic systems for essential thrombocythaemia and polycythaemia vera. British journal of haematology. 2020;189(2):291-302.

17. Tefferi A, Lasho TL, Guglielmelli P, et al. Targeted deep sequencing in polycythemia vera and essential thrombocythemia. Blood advances. 2016;1(1):21-30.

18. Lasho TL, Mudireddy M, Finke CM, et al. Targeted next-generation sequencing in blast phase myeloproliferative neoplasms. Blood advances. 2018;2(4):370-380.

19. Passamonti F. Stem cell transplant in MF: it's time to personalize. Blood, The Journal of the American Society of Hematology. 2019;133(20):2118-2120.

20. Barbui T, Thiele J, Ferrari A, Vannucchi AM, Tefferi A. The new WHO classification for essential thrombocythemia calls for revision of available evidences. Blood cancer journal. 2020;10(2):1-6.

21. Grinfeld J, Nangalia J, Baxter EJ, et al. Classification and personalized prognosis in myeloproliferative neoplasms. New England Journal of Medicine. 2018;379(15):1416-1430.

22. Swerdlow SH CE, Harris NL, Jaffe ES, Pileri SA, Stein H, Thiele J. WHO classification of tumours of haematopoietic and lymphoid tissues. . 2017; Volume 2:104-105. 
23. Lee J-M, Kim Y-J, Park S-S, Han E, Kim M, Kim Y. Simultaneous Monitoring of Mutation and Chimerism Using Next-Generation Sequencing in Myelodysplastic Syndrome. Journal of Clinical Medicine. 2019;8(12):2077.

24. Li MM, Datto M, Duncavage EJ, et al. Standards and guidelines for the interpretation and reporting of sequence variants in cancer: a joint consensus recommendation of the Association for Molecular Pathology, American Society of Clinical Oncology, and College of American Pathologists. The Journal of molecular diagnostics. 2017;19(1):4-23.

25. McGowan-Jordan J SA, Schmid M. eds.C. ISCN : an international system for human cytogenomic nomenclature (2016). Basel: Karger; 2016.

26. Krzywinski M, Schein J, Birol I, et al. Circos: an information aesthetic for comparative genomics. Genome research. 2009;19(9):1639-1645.

27. Tefferi A, Guglielmelli P, Lasho TL, et al. MIPSS70+ Version 2.0: Mutation and Karyotype-Enhanced International Prognostic Scoring System for Primary Myelofibrosis. Journal of clinical oncology: official journal of the American Society of Clinical Oncology. 2018;36(17):1769.

28. Andréasson B, Pettersson $H$, Wasslavik C, Johansson P, Palmqvist L, Asp J. ASXL1 mutations, previous vascular complications and age at diagnosis predict survival in $85 \mathrm{WHO}$-defined polycythaemia vera patients. British Journal of Haematology. 2020.

29. Tefferi A, Pardanani A. Mutations and prognosis in myeloproliferative neoplasms. Leukemia \& lymphoma. 2019;60(5):1112-1113.

30. Jeromin S, Haferlach T, Grossmann V, et al. High frequencies of SF3B1 and JAK2 mutations in refractory anemia with ring sideroblasts associated with marked thrombocytosis strengthen the assignment to the category of myelodysplastic/myeloproliferative neoplasms. Haematologica. 2013;98(2):e15.

31. Broséus J, Alpermann T, Wulfert M, et al. Age, JAK2 V617F and SF3B1 mutations are the main predicting factors for survival in refractory anaemia with ring sideroblasts and marked thrombocytosis. Leukemia. 2013;27(9):1826-1831.

32. Tefferi A, Nicolosi $M$, Mudireddy $M$, et al. Revised cytogenetic risk stratification in primary myelofibrosis: analysis based on 1002 informative patients. Leukemia. 2018;32(5):1189-1199.

33. Tefferi A, Lasho T, Abdel-Wahab O, et al. IDH1 and IDH2 mutation studies in 1473 patients with chronic-, fibrotic-or blast-phase essential thrombocythemia, polycythemia vera or myelofibrosis. Leukemia. 2010;24(7):1302-1309.

34. McKenney AS, Lau AN, Somasundara AVH, et al. JAK2/IDH-mutant-driven myeloproliferative neoplasm is sensitive to combined targeted inhibition. The Journal of clinical investigation. 2018;128(2):789-804.

35. Tefferi A, Lasho TL, Finke CM, et al. Targeted deep sequencing in primary myelofibrosis. Blood advances. 2016;1(2):105-111.

36. Vannucchi A, Lasho T, Guglielmelli P, et al. Mutations and prognosis in primary myelofibrosis. Leukemia. 2013;27(9):1861-1869. 
37. Moran-Crusio K, Reavie L, Shih A, et al. Tet2 loss leads to increased hematopoietic stem cell selfrenewal and myeloid transformation. Cancer cell. 2011;20(1):11-24.

38. Lundberg P, Karow A, Nienhold R, et al. Clonal evolution and clinical correlates of somatic mutations in myeloproliferative neoplasms. Blood, The Journal of the American Society of Hematology. 2014;123(14):2220-2228.

39. Tefferi A, Lasho TL, Hanson CA, Ketterling RP, Gangat N. Screening for ASXL1 and SRSF2 mutations is imperative for treatment decision-making in otherwise low or intermediate-1 risk patients with myelofibrosis. British Journal of Haematology.2018;183(4):678-681.

\section{Tables}

Table 1. Overall characteristics of patients in this study

\begin{tabular}{|c|c|c|c|c|c|c|}
\hline Variables & $\begin{array}{l}\text { Total, } \\
\mathrm{N}=200\end{array}$ & $\mathrm{PV}, \mathrm{N}=55$ & $\mathrm{ET}, \mathrm{N}=70$ & $\mathrm{PMF}, \mathrm{N}=66$ & $\begin{array}{c}\text { Other } \\
\text { MPN }^{\mathrm{a}}, \mathrm{N}=9\end{array}$ & $P$ \\
\hline Age at diagnosis, mean $\pm S D$ & $49.0 \pm 14.7$ & $50.3 \pm 12.3$ & $43.2 \pm 15.3$ & $52.5 \pm 13.6$ & $61.4 \pm 14.2$ & $<0.001$ \\
\hline Sex, male (\%) & $\begin{array}{c}43.0 \\
(86 / 200)\end{array}$ & $52.7(29 / 55)$ & $35.7(25 / 70)$ & $39.4(26 / 66)$ & $66.7(6 / 9)$ & 0.109 \\
\hline $\begin{array}{l}\text { Follow-up months, } \\
\text { mean } \pm \text { SD }\end{array}$ & $64.7 \pm 69.4$ & $76.4 \pm 67.7$ & $78.5 \pm 73.8$ & $46.0 \pm 64.8$ & $23.6 \pm 20.2$ & $<0.001$ \\
\hline \multicolumn{7}{|l|}{ Blood cell count } \\
\hline $\begin{array}{l}\text { White blood cells }\left(10^{9} / 1\right) \text {, } \\
\text { mean } \pm \text { SD }\end{array}$ & $12.9 \pm 13.7$ & $12.9 \pm 7.4$ & $9.5 \pm 5.5$ & $11.8 \pm 11.9$ & $47.8 \pm 37.3$ & $<0.001$ \\
\hline $\begin{array}{l}\text { Hemoglobin }(\mathrm{g} / \mathrm{l}) \\
\text { mean } \pm \text { SD }\end{array}$ & $129.3 \pm 33.6$ & $164.7 \pm 31.3$ & $126.2 \pm 20.6$ & $107.7 \pm 21.3$ & $95.3 \pm 18.7$ & $<0.001$ \\
\hline Platelet $\left(10^{9} / 1\right)$, mean \pm SD & $560.5 \pm 484.8$ & $542.2 \pm 324.9$ & $803.7 \pm 608.7$ & $367.5 \pm 340.3$ & $197.2 \pm 147.5$ & $<0.001$ \\
\hline Bone marrow fibrosis, \% & $\begin{array}{c}46.0 \\
(92 / 200)\end{array}$ & $16.4(9 / 55)$ & $21.4(15 / 70)$ & $\begin{array}{l}100.0 \\
(66 / 66)\end{array}$ & $22.2(2 / 9)$ & 0.064 \\
\hline Splenomegaly, \% & $\begin{array}{c}31.5 \\
(63 / 200)\end{array}$ & $27.3(15 / 55)$ & $11.4(8 / 70)$ & $54.5(36 / 66)$ & $44.4(4 / 9)$ & 0.364 \\
\hline Vascular event ${ }^{b}, \%$ & $\begin{array}{c}20.0 \\
(40 / 200)\end{array}$ & $\begin{array}{c}36.4 \\
(20 / 55)\end{array}$ & $\begin{array}{c}20.0 \\
(14 / 70)\end{array}$ & $9.1(6 / 66)$ & $0(0 / 9)$ & 0.275 \\
\hline Abnormal karyotype ${ }^{c}, \%$ & $\begin{array}{c}22.5 \\
(38 / 169)\end{array}$ & $18.6(8 / 43)$ & $9.8(6 / 61)$ & $37.5(21 / 56)$ & $33.3(3 / 9)$ & 0.003 \\
\hline Complex karyotype ${ }^{\mathrm{c}}, \%$ & $4.7(8 / 169)$ & $4.7(2 / 43)$ & $3.3(2 / 61)$ & $7.1(4 / 56)$ & $0(0 / 9)$ & 0.692 \\
\hline $\begin{array}{l}\text { Number of mutations, } \\
\text { mean } \pm \text { SD }\end{array}$ & $1.3 \pm 1.0$ & $1.4 \pm 0.9$ & $1.1 \pm 0.9$ & $1.6 \pm 1.1$ & $0.9 \pm 0.9$ & 0.024 \\
\hline
\end{tabular}

$P V$ polycythemia vera, ET essential thrombocythemia, $P M F$ primary myelofibrosis, $M P N$ myeloproliferative neoplasm, $C N L$ chronic neutrophilic leukemia, $S D$ standard deviation.

Bold indicates significant values with $P<0.05$

Group comparisons were performed using the Kruskal-Wallis and Chi-square test for continuous and categorical variables, respectively.

${ }^{\mathrm{a}}$ Other MPN includes CNL $(\mathrm{n}=4)$ and MPN-unclassifiable $(\mathrm{n}=5)$

bVascular event includes thrombosis history at diagnosis and related microcirculation symptoms such as headaches, lightheadedness, atypical chest pain, transient visual disturbances, and acral paresthesias

'Total of 169 BM karyotypes were available. 
Table 2. Proportion of patients possessing clonal genetic marker after applying sequential criteria for genetic aberrations.

\begin{tabular}{|c|c|c|c|c|c|}
\hline & PV & ET & $\overline{P M F}$ & $3 \mathrm{MPNs}^{\mathrm{a}}$ & $\begin{array}{l}\text { Other } \\
\text { MPN }^{\mathrm{b}}\end{array}$ \\
\hline Case number & 55 & 70 & 66 & 191 & 9 \\
\hline Triple mutations ${ }^{\mathrm{c}}$ & $\begin{array}{c}49 \\
(89.1 \%)\end{array}$ & $\begin{array}{c}51 \\
(72.9 \%)\end{array}$ & $\begin{array}{c}46 \\
(69.7 \%)\end{array}$ & $\begin{array}{c}146 \\
(76.4 \%)\end{array}$ & $2(22.2 \%)$ \\
\hline Any of seven mutations ${ }^{d}$ & $0(0 \%)$ & $2(2.9 \%)$ & $6(9.1 \%)$ & $8(4.2 \%)$ & $3(33.3 \%)$ \\
\hline $\begin{array}{l}\text { Other mutations }{ }^{\mathrm{e}} \text { and/or abnormal } \\
\text { karyotype }\end{array}$ & $1(1.8 \%)$ & $1(1.4 \%)$ & $9(13.6 \%)$ & $11(5.8 \%)$ & $4(22.4 \%)$ \\
\hline Any clonal genetic marker ${ }^{f}$ & $\begin{array}{c}50 \\
(90.9 \%)\end{array}$ & $\begin{array}{c}54 \\
(77.1 \%)\end{array}$ & $\begin{array}{c}61 \\
(92.4 \%)\end{array}$ & $\begin{array}{c}165 \\
(86.4 \%)\end{array}$ & $7(77.8 \%)$ \\
\hline All negative & $5(9.1 \%)$ & $\begin{array}{c}16 \\
(22.9 \%)\end{array}$ & $5(7.6 \%)$ & $26(13.6 \%)$ & $2(22.2 \%)$ \\
\hline
\end{tabular}

PV polycythemia vera, ET essential thrombocythemia, PMF primary myelofibrosis, MPN myeloproliferative neoplasm, CNL chronic neutrophilic leukemia.

${ }^{a} \mathrm{PV}, \mathrm{ET}$ and PMF, ${ }^{\mathrm{b}} \mathrm{CNL}(\mathrm{n}=4)$ and MPN-unclassifiable $(\mathrm{n}=5),{ }^{\mathrm{c} J A K 2}$, CALR and MPL for 3 MPNs and CSF3R for CNL, dany of mutations including ASXL1, EZH2, TET2, IDH1/2, SRSF2, SF3B1 among triple negative patients, eany mutation other than the above-mentioned mutations, fany mutation and/or abnormal karyotype.

Table 3. Multivariate analysis of clinical and genetic factors for overall survival, leukemic transformation, and fibrosis progression

\begin{tabular}{|c|c|c|c|c|c|c|c|c|c|}
\hline \multirow[t]{2}{*}{ Variables } & \multicolumn{3}{|c|}{ Overall survival } & \multicolumn{3}{|c|}{ Leukemic transformation } & \multicolumn{3}{|c|}{ Fibrotic progression } \\
\hline & $P$ & HR & $95 \% \mathrm{CI}$ & $P$ & HR & $95 \% \mathrm{CI}$ & $P$ & HR & $95 \% \mathrm{CI}$ \\
\hline $\begin{array}{l}\text { Diagnosis }^{\mathrm{a}} \\
\text { PB blasts (\%) }\end{array}$ & 0.0087 & 78.2 & $3.0-2027.3$ & 0.0486 & 1.3 & $1.0-1.6$ & & & \\
\hline No. mutation & & & & & & & 0.0352 & 2.0 & $1.1-4.0$ \\
\hline$A S X L 1$ & & & & & & & 0.0358 & 4.3 & $1.1-16.4$ \\
\hline$R U N X 1$ & & & & 0.005 & 68.1 & $3.6-1300.4$ & & & \\
\hline SF3B1 & & & & & & & 0.0009 & 31.5 & 4.1-243.3 \\
\hline TP53 & 0.0041 & 64.2 & 3.8-1096.5 & 0.0364 & 16.3 & $1.2-222.7$ & & & \\
\hline IDH1/2 & & & & 0.0051 & 32.5 & 2.8-371.1 & 0.0011 & 21.2 & $3.4-132.2$ \\
\hline$-7 / \operatorname{del}(7 q)$ & 0.0219 & 14.0 & $1.5-132.7$ & & & & & & \\
\hline $\operatorname{Del}(20 q)$ & & & & & & & 0.0002 & 44.5 & $6.1-323.0$ \\
\hline
\end{tabular}

$H R$ hazard ratio, $C I$ confidence interval, $P B$ peripheral blood, No.number.

Variables assigned to be significant prognostic factors in univariate analysis were included in the multivariate analysis. The fibrosis progression was analyzed among patients with polycythemia vera and essential 
thrombocythemia, excluding primary myelofibrosis.

${ }^{a}$ Diagnosis of primary myelofibrosis.

Table 4. Comparison of risk groups in patients with PV and ET.

\begin{tabular}{lcccc}
\hline & Low & Intermediate & High & $P$ (contingency coefficient) \\
\hline $\begin{array}{l}\text { PV } \\
\text { MIPSS PV }\end{array}$ & 18 & 23 & 14 & $<0.001(0.514)$ \\
$\quad$ Low & 18 & 14 & 6 & \\
\hline Intermediate & 0 & 9 & 5 & \\
\hline High & 0 & 0 & 3 & \\
\hline ET & 42 & 24 & 4 & $<0.001(0.554)$ \\
MIPSS ET & & & & \\
\hline Low & 41 & 19 & 0 & \\
\hline Intermediate & 1 & 3 & 2 & \\
\hline High & 0 & 2 & 2 & \\
\hline
\end{tabular}

MIPSS Mutation-enhanced International Prognostic Scoring System, $P V$ polycythemia vera, ET essential thrombocythemia.

Risk factors for PV: advanced age ( $\geq 67$ years, 5 points ; 57-66 years, 2 points), leukocytosis ( $\geq 15 \times 10^{9} / 1,1$ point), and presence of a vascular event (1 point); low risk (no risk factors), intermediate risk (1-2 points), high ( $\geq 3$ points).

Risk factors for ET: advanced age ( $\geq 60$ years, 2 points), leukocytosis ( $\geq 11 \times 10^{9} / 1,1$ point), and presence of vascular event (1 point); low risk (no risk factors), intermediate risk (1-2 points), high (3-4 points).

Table 5. Comparison of risk groups in PMF patients.

\begin{tabular}{lccccc}
\hline DIPSS & Low & Intermediate-1 & Intermediate-2 & High & $P$ (Contingency coefficient) \\
\hline MIPSS70 & & & & & $<0.001(0.620)$ \\
Low & 15 & 1 & 0 & 0 & \\
\hline Intermediate & 8 & 12 & 11 & 0 & \\
\hline High & 2 & 3 & 10 & 4 & \multirow{2}{*}{$<0.001(0.603)$} \\
MIPSS70+ & & & & & \\
\hline Very low & 5 & 1 & 0 & 0 & \\
\hline Low & 10 & 5 & 2 & 0 & \\
\hline Intermediate & 4 & 4 & 3 & 0 & \\
\hline High & 2 & 3 & 8 & 1 & \\
\hline Very High & 0 & 0 & 6 & 2 & \\
\hline
\end{tabular}

DIPSS Dynamic International Prognostic Scoring System, MIPSS70 Mutation-enhanced International prognostic scoring system, MIPSS70+ Karyotype-enhanced MIPSS70. 
A

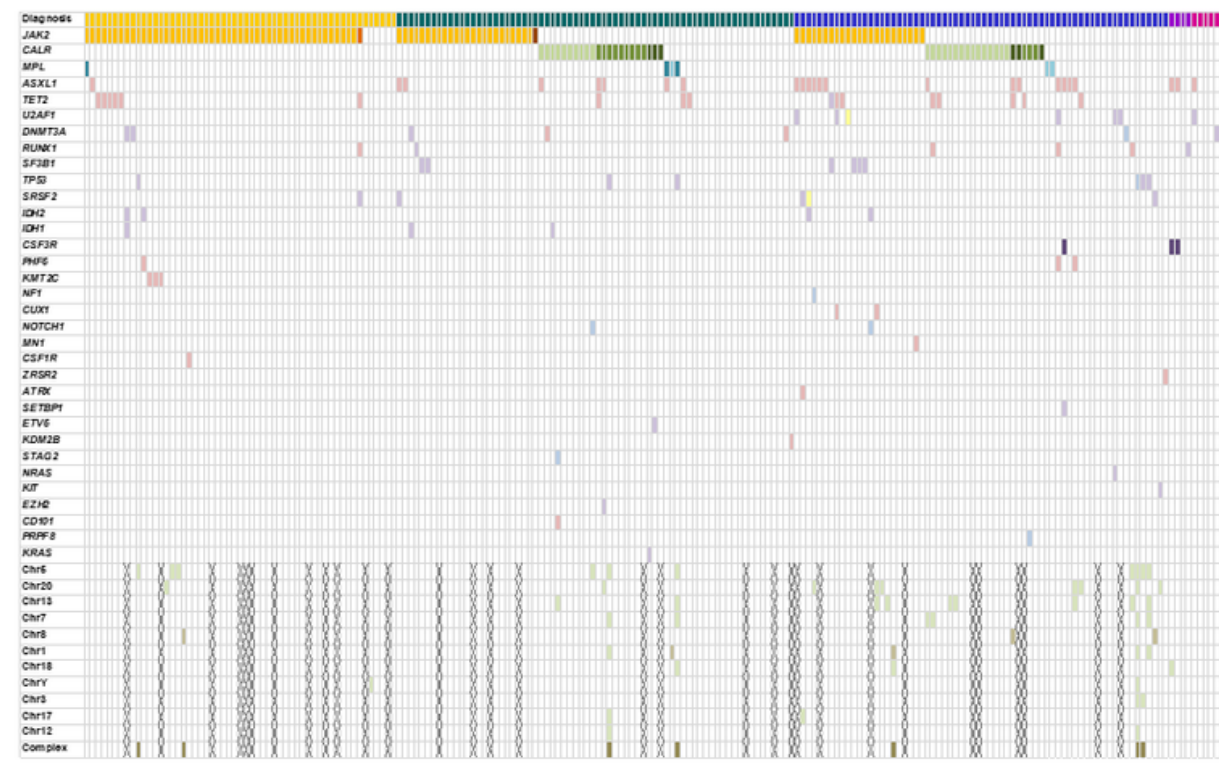

$B^{100}$
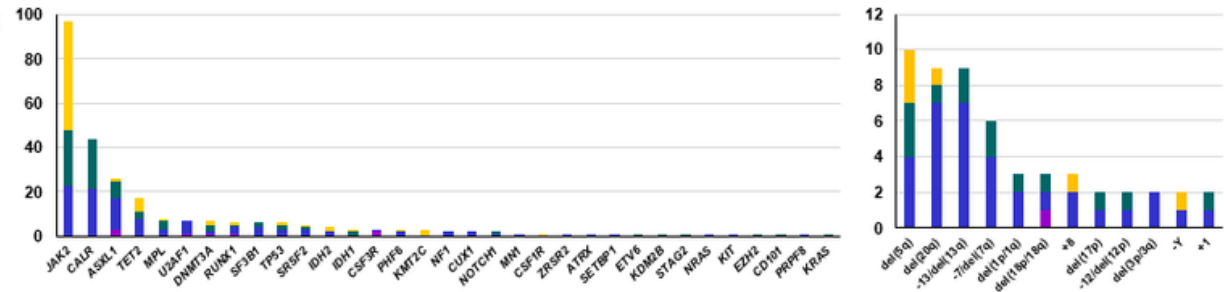

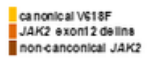

CALR VDO 1
CALR

noncanonical CALR

$\mid$ canconical MPL
noncanonical MPL

Ileanonical CSFIR

mimsonses mutation
truncation mutation

Intarem muthton
intronic mutation

lioun

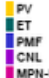

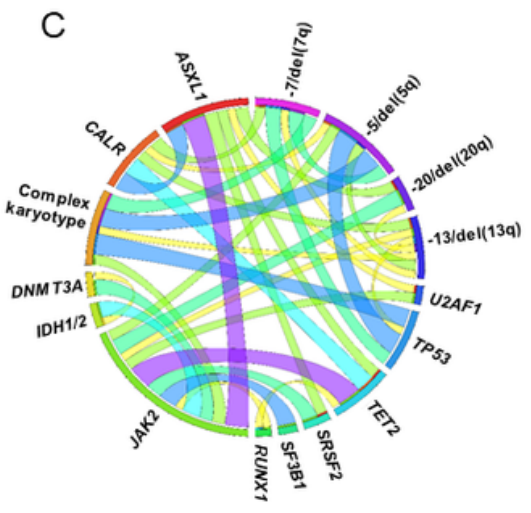

\section{Figure 1}

Genomic landscapes of 200 MPN patients (A) The mosaic plot and (B) bar-charts of mutations and chromosomal abnormalities (C) The circos plot indicating recurrent co-occurrences of genetic abberations by ribbon widths. PV Polycythemia vera, ET essential thrombocythemia, PMF primary myelofibrosis, CNL chronic neutrophilic leukemia, MPN-U, MPN-unclassifiable, MPN myeloproliferative neoplasm 
A

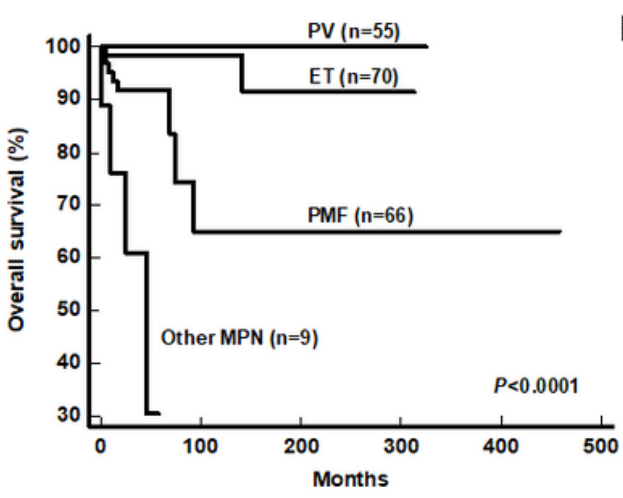

C

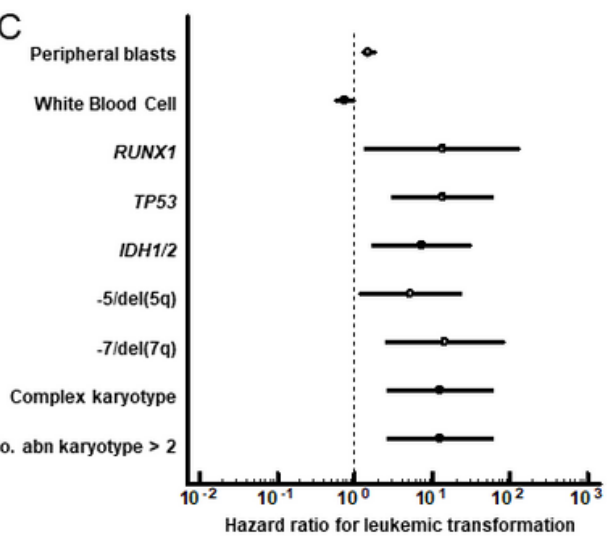

B

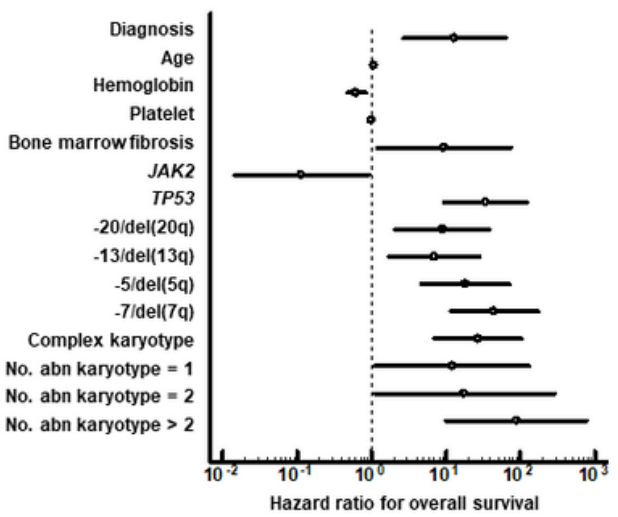

D

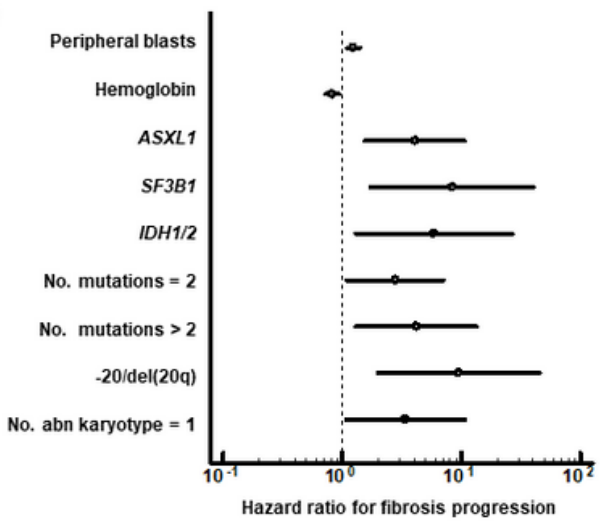

Figure 2

(A) Overall survival curves of the patients according to disease category. (B) Hazard ratios of significant factors for overall survival and (C) leukemic transformation in PV, ET, PMF and (D) fibrosis progression in PV and ET patients. PV Polycythemia vera, ET essential thrombocythemia, PMF primary myelofibrosis, MPN myeloproliferative neoplasm, No number, abn abnormal.

\section{Supplementary Files}

This is a list of supplementary files associated with this preprint. Click to download.

- Supplementarytablefigureexphematoloncol.docx 